\title{
Retrieval-induced forgetting occurs in tests of item recognition
}

\author{
JASON L. HICKS and JEFFREY J. STARNS \\ Louisiana State University, Baton Rouge, Louisiana
}

\begin{abstract}
Using the retrieval-practice paradigm (Anderson, R. A. Bjork, \& E. L. Bjork, 1994), we tested whether or not retrieval-induced forgetting could be found in item recognition tests. In Experiment 1, retrieval practice on items from semantic categories depressed recognition of nonpracticed items from the same categories. Similar results were found in Experiment 2 in a more stringent source test for practiced, nonpracticed, and new items. These results conceptually replicate those of previous retrievalinduced forgetting studies done with cued recall (e.g., Anderson et al., 1994). Our findings are inconsistent with the hypothesis that item-specific cues during retrieval will eliminate retrievalinterference in the retrieval-practiceparadigm (Butler, Williams, Zacks, \& Maki, 2001). We discuss our results in relation to other retrieval interference and inhibition effects in recall and recognition.
\end{abstract}

Retrieval-induced forgetting refers to the phenomenon in which retrieval of some studied items causes temporary inaccessibility of other studied items when both types are associated with a common retrieval cue (e.g., Anderson, R. A. Bjork, \& E. L. Bjork, 1994; Roediger, 1974; Watkins, 1978). Anderson and his colleagues have demonstrated that this form of retrieval impairment has an effect in episodic memory across retrieval sessions (e.g., Anderson et al., 1994). That is, retrieval of some items at one point in time has a detrimental effect on retrieval of other items in a later retrieval session. Anderson and colleagues' recent work has typically relied on preexisting associations between semantic or conceptual categories. However, other work demonstrates that competition among items that are associated with nonsemantic cues can also cause retrieval-induced forgetting, be they perceptual or spatial cues (Ciranni \& Shimamura, 1999) or spatiotemporal cues (Koutstaal, Schacter, Johnson, \& Galluccio, 1999).

Because the experiments in our study relied on preexisting associations among category exemplars, we will describe next the standard paradigm used to demonstrate retrieval-induced forgetting. Using a retrieval-practice paradigm, Anderson et al. (1994) showed that retrieving some category exemplars from episodic memory prior to a final cued recall test later caused forgetting of other studied exemplars from the same category. In the first phase of the paradigm, people learn several category-

We thank Joanna Arrington, Angela Roan, Cristine Roussel, and Matt Sentilles for their dedicated help in collecting the data, and Lisa Sheppard-Goodlett for her help in scoring the retrieval practice data. We also thank Mike Anderson, Doug Nelson, and Roddy Roediger for their comments on an earlier version of the manuscript. Correspondence concerning this article should be addressed to J. L. Hicks, Department of Psychology, Louisiana State University, Baton Rouge, LA 70803-5501 (e-mail: jhicks@1su.edu). exemplar pairs (e.g., food-cherry) from multiple categories (e.g., food, colors, etc.). In the second phase, retrieval practice occurs for some of the studied exemplars, using cued recall. Exemplars are usually cued with the category label and the first letter or two of the exemplar (e.g., food-ch ). Critical to this practice phase is that only some categories are practiced, and within those categories, only some of the exemplars are practiced. By convention, practiced categories are labeled $\mathrm{Rp}$, items from these categories that were individually practiced are labeled $\mathrm{Rp}+$, nonpracticed items from the same practiced categories are labeled $\mathrm{Rp}-$, and items from nonpracticed categories are labeled Nrp.

In the final test phase, all learned exemplars are tested in a surprise cued recall test that might use the category label (e.g., food) or a category-plus-stem cue (e.g., foodch_____; Anderson et al., 1994). The standard finding in this paradigm is that $\mathrm{Rp}+$ items are recalled better and $\mathrm{Rp}-$ items are recalled worse in comparison with the Nrp baseline. That is, retrieval practice facilitates later recall of $R p+$ items but impairs recall of $R p-i t e m s$. The prevailing theoretical view is that the temporary forgetting of $\mathrm{Rp}-$ items is caused by their inhibition during the intermediate retrieval practice phase. As $\mathrm{Rp}+$ items receive retrieval practice, competing $\mathrm{Rp}-$ items are ostensibly inhibited to reduce their interference with target item retrieval, thus causing their temporary inaccessibility in the final memory test (Anderson et al., 1994).

In contrast to most studies already reported, the present study explores whether or not retrieval-induced forgetting can be found in the context of a final recognition test as opposed to the recall tests that have traditionally been used. To our knowledge, there is only one published attempt that examined retrieval-induced forgetting in a recognition test (Koutstaal et al., 1999). In that study, retrievalinduced forgetting was found in a free recall test (Experiment 1), but not in a recognition test (Experiment 2). 
Koutstaal et al. had people physically perform activities during the encoding session (e.g., "trace the boomerang"). Two days following the encoding session, photographs of actors performing half of the same activities were given for review ( $\mathrm{Rp}+$ items). The participants were asked to use the photographs to reconstruct the entire encoding context as best as possible during this photograph review. A between-subjects control group that did not engage in photograph review was used as the nonpracticed baseline (i.e., the Nrp condition).

At test, all participants were asked either to free recall the entire set of performed actions (Experiment 1) or to recognize objects involved in the performed actions (Experiment 2). Significant facilitation was found for $\mathrm{Rp}+$ actions in both retrieval tasks. Only in the free recall test, however, was impairment of Rp- actions found. Koutstaal et al. (1999) concluded that retrieval-induced forgetting is unlikely to be found in memory tests that reinstate powerful contextual cues for individual items. Given that recognition tests typically present identical cues of the studied material, one could consider recognition as the most difficult paradigm in which to find impaired retrieval of Rp-items. Anderson and R. A. Bjork (1994) concurred by predicting that evidence of retrieval inhibition might be more difficult to find in recognition tests. In agreement with these findings, Butler, Williams, Zacks, and Maki (2001) found significant retrievalinduced forgetting in a category cued recall test, but not in tests of memory for which item-specific cues were supplied (e.g., word fragment cued recall and word fragment completion). Other paradigms also suggest that evidence of retrieval inhibition in measures of accuracy is difficult to find in a recognition test. One example is the directed forgetting literature (see MacLeod, 1999, for a discussion), and another is the part-set cuing literature (Slamecka, 1975; Todres \& Watkins, 1981). Although part-set cuing effects have been found in recognition latencies under certain conditions (e.g., Neely, Schmidt, \& Roediger, 1983), such effects have not generalized to recognition accuracy.

Despite a previous failure to find retrieval-induced forgetting in recognition memory (Koutstaal et al., 1999), we believe that there are important reasons to suspect that retrieval-induced forgetting could nonetheless be found in a recognition test. Theoretically, if retrievalinduced forgetting is attributable to inhibition of an $\mathrm{Rp}-$ item's representation (Anderson et al., 1994; Anderson $\&$ Spellman, 1995), then the effect should be obtained in recognition because the availability of the item is directly affected. In contrast, interference theories that rely on strength-dependent competition mechanisms (see, e.g., McGeoch, 1942; Raaijmakers \& Shiffrin, 1981) would predict no retrieval-induced forgetting in a recognition test. For example, strengthening some items in a list makes nonstrengthened items less accessible in recall in comparison with lists where no items are strengthened, but this effect disappears in recognition (e.g., Ratcliff,
Clark, \& Shiffrin, 1990). Even an alternative view of classbased inhibition known as response-set suppression predicts significant impairment of the entire class of suppressed (i.e., $\mathrm{Rp}-$ ) items on tests of recall but not on tests of recognition because making previously suppressed responses available circumvents the lack of accessibility found in recall (Postman \& Stark, 1969). Thus, the view that individual $\mathrm{Rp}$ - item representations are affected directly by inhibition (see, e.g., Anderson \& Spellman, 1995) makes the unique prediction of impairment in any explicit test of memory, including recognition tests.

Empirically, Koutstaal et al.'s (1999) finding of retrievalinduced forgetting in free recall was demonstrated with a novel spatiotemporal context as the organizing link among the encoded actions. This procedure contrasts with Anderson et al.'s (1994) original paradigm in which the organizing principle was a very well-learned conceptual category. Consequently, we felt it important to attempt to demonstrate retrieval-induced forgetting using a standard recognition memory paradigm patterned more closely after the original demonstrations of the effect.

\section{EXPERIMENTS 1 AND 2}

Following the standard methods, we had three general phases in our experiments: encoding of category exemplars, retrieval practice for half of the items from half of the categories, and final retrieval of all studied items following a brief delay. The only significant deviations from the standard procedure were that individual exemplars, rather than category-exemplar pairs, were initially studied in order to minimize contextual cues associated with encoding, and that item recognition was used as the final retrieval task.

Based on the existing theoretical analysis, the empirical predictions were relatively straightforward. Assuming that retrieval practice decreases the episodic availability of $\mathrm{Rp}$ - items, recognition should be worse for these items relative to the Nrp baseline. This outcome would conceptually replicate the existing literature that heretofore has demonstrated the effect only in free or cued recall. However, an alternative possibility is that the copy cues offered in item recognition will provide direct access to an item's episodic representation. As such, retrieval-induced forgetting may not be found in recognition, which would be consistent with both Koutstaal et al.'s (1999) null result and Butler et al.'s (2001) failure to find the effect in itemspecific tests.

In Experiment 2, we explored whether or not the effect would generalize to conditions in which people were asked to scrutinize their memories more carefully. Given that RP + items are strengthened in a different temporal context from that for the original study list, people may be able to use temporal cues as a way to determine when certain items have been encountered. In particular, making people explicitly consider that test items might have been studied, but not practiced, should produce a more care- 
ful decision. Research indicates that asking people to consider the source of their memories can reduce errors (e.g., Lindsay \& Johnson, 1989). To this end, we asked people to label test items as studied and practiced, studied but not practiced, or brand new on a source test. If the consequence of retrieval practice is to weaken the familiarity or strength of nonpracticed category members through inhibition, then such items may still be called new regardless of the more stringent source-monitoring test format. However, if the source test enables people to recover contextual cues from the original context of the study list that might otherwise go unexamined in a standard recognition test, then items otherwise inhibited by retrieval practice may be correctly attributed to their proper source (i.e., their inhibition is either released or circumvented).

\section{Method}

Participants. In each experiment, 40 undergraduate students participated for course extra credit. The participants were tested individually in sessions that lasted approximately $35 \mathrm{~min}$.

Materials. The primary stimuli consisted of 12 exemplars from each of eight target categories selected from the Battig and Montague (1969) category norms. The categories chosen were metals, animals, colors, body parts, fruits, weapons, professions, and instruments. Exemplars were generally the 12 normatively most frequent items in the category. For each category, two counterbalancing sets of 6 items each were created and used an equal number of times as targets versus lures on the recognition test. Additionally, 4 exemplars from each of the categories precious stones, clothing, and tools were used as buffer items in the study list, for a total of 12 buffer items. Six buffer items were shown at the beginning of the study list, and 6 were shown at the end.

Four of the eight categories were given subsequent retrieval practice $(\mathrm{Rp})$, whereas the remaining four categories were designated as nonpracticed (Nrp). Categories were designated as Rp versus Nrp an equal number of times across participants in accordance with complete counterbalancing. For the Rp categories, three of the studied items were practiced $(\mathrm{Rp}+)$, and the other three were not $(\mathrm{Rp}-)$. The practiced and unpracticed exemplars were counterbalanced as well. Small booklets were created to present category-stem cues for the retrieval practice phase. The 12 items designated for retrieval practice were included in addition to 3 items from each of the filler categories clothing and tools. The 18 items were ordered within the booklets in such a way that no 2 items from a given category were practiced in succession. Retrieval cues were printed on each page in a category-stem format (e.g., ANIMAL-MO___ Each participant used two identical booklets (one for each round of retrieval practice).

The 12 items from each category were used in the recognition test. One random order of the test was created under the constraints that (1) items from a given category were at least 5 items apart, (2) approximately the same number of old and new items were shown in the first 48 and last 48 test trials, and (3) no two RP+ items from a given category were ever tested before at least one $\mathrm{RP}-$ item from the same category was tested, and vice versa. Six of the 12 buffer items from the study list were tested in Positions 1-6 before any items of true interest were tested.

Procedure. The participants were informed that they would be studying a list of words for an unspecified memory test. A computer program created with SuperLab Pro (Cedrus Corporation, 1999) was used to present the study list. Each item was shown for $1.75 \mathrm{sec}$, followed by a $500-\mathrm{msec}$ interstimulus interval. After the study phase, the participants solved multiplication problems for $2 \mathrm{~min}$. Following this distractor task, the experimenter provided the participants with a retrieval practice booklet and explained that the cues should be used to recall studied words. Six seconds were provided for recall of each item. If no word came to mind before time elapsed, the participants were asked to leave the stem blank and turn the page. When the first packet was complete, the participants worked on math problems for another $2 \mathrm{~min}$ and then repeated the retrieval practice phase on an identical booklet (i.e., a total of two practice rounds were given). Following this second round of retrieval practice, the participants were asked to work on visual puzzles for $10 \mathrm{~min}$, after which the recognition test was administered. In Experiment 1, the participants were given individual items to consider for an old-new decision (i.e., category cues were not used at test). Each word remained on the computer screen until a recognition response was made. In Experiment 2, the participants were asked to indicate whether test items were studied and practiced ("P"), studied but not practiced ("NP"), or new.

\section{Results}

In Experiment 1, the probability of correct recall from the first and second rounds of retrieval practice was .77 $(S E=.03)$ and $.83(S E=.02)$, respectively. In Experiment 2, these probabilities were $.79(S E=.02)$ and .84 $(S E=.02)$, respectively. These data demonstrate that retrieval practice was largely successful. In what follows, hit and false alarm rates for each item of interest $(\mathrm{Rp}+$, $\mathrm{Nrp}, \mathrm{Rp}-$ ) are reported first for each experiment. Data and analyses from the source test in Experiment 2 are presented last. The probability of a Type I error was set at .05 for all analyses, and a Bonferroni correction was applied to all pairwise comparisons.

Recognition accuracy. Table 1 presents the hit and false alarm rates. Not surprisingly, $\mathrm{Rp}+$ items greatly benefited from retrieval practice, producing a hit rate approximately $20 \%$ higher than that for Nrp items. In contrast, the hit rate for $\mathrm{Rp}-$ items was lower than that for Nrp items by approximately $9 \%$. A one-way within-subjects analysis of variance confirmed these overall differences $\left[F(2,78)=74.98, M S_{\mathrm{e}}=.01\right.$, for Experiment $1 ; F(2,78)=$

Table 1

Probability of Positive Recognition Responses to Targets and Lures by Retrieval Practice Condition in Experiments 1 and 2

\begin{tabular}{|c|c|c|c|c|}
\hline \multirow[b]{2}{*}{ Condition } & \multicolumn{2}{|c|}{ Targets } & \multicolumn{2}{|c|}{ Lures } \\
\hline & $M$ & $S E$ & $M$ & $S E$ \\
\hline \multicolumn{5}{|c|}{ Experiment 1} \\
\hline $\mathrm{Rp}+$ & .91 & .02 & .17 & .02 \\
\hline Nrp & .71 & .02 & .18 & .02 \\
\hline $\mathrm{Rp}-$ & .62 & .02 & .17 & .02 \\
\hline \multicolumn{5}{|c|}{ Experiment $2 *$} \\
\hline $\mathrm{Rp}+$ & .94 & .01 & .18 & .02 \\
\hline Nrp & .74 & .02 & .19 & .02 \\
\hline $\mathrm{Rp}-$ & .65 & .02 & .18 & .02 \\
\hline
\end{tabular}

$\overline{\text { Note- }-\mathrm{Rp}+\text {, items from practiced categories that were individually }}$ practiced; Nrp, items from nonpracticed categories; $\mathrm{Rp}-$, items from practiced categories that were not individually practiced. The false alarm rate to lures for $\mathrm{Rp}+$ and $\mathrm{Rp}-$ items represent a common proportion (i.e., lures from practiced categories). *In Experiment 2, recognition probabilities were inferred from the source claims. 
$66.32, M S_{\mathrm{e}}=.01$, for Experiment 2]. Pairwise comparisons revealed that the hit rate for $\mathrm{Rp}+$ items was greater than that for either Nrp items or Rp- items, reflecting the beneficial effects of retrieval practice [lesser $t(39)=$ 9.0 for Experiment 1; lesser $t(39)=7.9$ for Experiment 2]. Of greater interest was that the hit rate for $\mathrm{Rp}-$ items was significantly lower than that for Nrp items, reflecting the detrimental effects of retrieval practice $[t(39)=3.8$ for Experiment $1 ; t(39)=3.5$ for Experiment 2]. ${ }^{1}$ A within-subjects comparison showed no significant difference between false alarms to items from $\mathrm{Rp}$ and Nrp categories $[t(39)<1.0$ for both experiments].

Source attributions. Table 2 presents the source claims given to each type of target and lure item for each retrieval practice condition in Experiment 2. The overall impression gleaned from this table is that people were generally good at the source task. Rp + items were mostly identified as practiced and were identified as not practiced more often than new. Nrp and Rp- items were rarely identified as practiced, and mostly as not practiced, with claims of new being intermediate. Lure items, when falsely recognized, were more often attributed to the weaker category of not practiced as opposed to being called practiced. All of these patterns replicate those ordinarily found in the source-monitoring literature for sources that differ in memorability (Marsh \& Hicks, 1998).

Of greater importance, however, were the claims given to Nrp and Rp - items. Correct attributions as "not practiced" were higher for Nrp than for Rp- items [t(39) = $3.27, S E=.03]$. The opposite effect was found for claims of "new" for these items, which is simply the complement of the inferred recognition analysis reported earlier. Misattributions of Nrp and Rp - items as "practiced" were low and not different from one another $[t(39)<1.0]$. The retrieval-induced forgetting in this experiment was therefore isolated to correct attributions of nonpracticed items as not practiced.

\section{Discussion}

In two experiments retrieval-induced forgetting was found in tests of item recognition. To our knowledge, this is the first published report of such a finding. Significantly worse recognition was found for $\mathrm{Rp}-$ items than for Nrp items, replicating the literature that has traditionally used cued recall to measure the resulting effects of retrieval practice (and nonpractice). In Experiment 2, the effect was conceptually replicated even though people had to very carefully determine the source of each test item in a modified recognition test. This experiment could be considered a "best-case" situation in which to eliminate the impairment of Rp - items, were it theoretically possible. Our results are inconsistent with Butler et al.'s (2001) finding that item-specific retrieval cues eliminate retrieval-induced forgetting. Our data support the conclusion that retrieval practice makes $\mathrm{Rp}-$ items less available to conscious retrieval, whether that retrieval consists of explicit recall or of recognition.

One issue to consider is whether or not methodological details are important in explaining our results. There is at least one unpublished dissertation report of a failure to find retrieval-induced forgetting in a recognition test (Lang, 2000). One distinguishing feature in this study was the use of traditional category-exemplar pair encoding, whereas we had people encode individual exemplars without a category cue. However, we can think of at least two reasons why this is likely not a critical factor. First, in other work in our lab (Starns \& Hicks, in press), we found retrieval-induced forgetting in recognition when people encoded theme-exemplar pairs (e.g., STRANDbrush). Second, there are other unpublished findings of retrieval-induced forgetting in recognition, most using the standard category-exemplar pair encoding paradigm (Anderson, 2003; Anderson, De Kok, \& Child, 1997). We therefore believe that our results are not unique to the encoding methodology that we used.

Our findings also suggest that Koutstaal et al.'s (1999) null result in recognition was probably due to factors other than the recognition test used to measure performance. One factor was that well-learned semantic associations were not used as a common retrieval cue. The general organizing link among the encoded actions in that study was the overall spatiotemporal context. A second mitigating factor could have been that the encoding context in the Koutstaal et al. study provided a rich set of memorial details that might have been accessible during recognition. That is, recollection of features of the en-

Table 2

Probability of Source Judgments to Targets and Lures by Retrieval Practice Condition in Experiment 2

\begin{tabular}{|c|c|c|c|c|c|c|c|c|c|c|}
\hline \multirow{3}{*}{$\begin{array}{l}\text { Source } \\
\text { Claim }\end{array}$} & \multicolumn{6}{|c|}{ Targets } & \multicolumn{4}{|c|}{ Lures } \\
\hline & \multicolumn{2}{|c|}{$\mathrm{Rp}+$} & \multicolumn{2}{|c|}{ Nrp } & \multicolumn{2}{|c|}{$\mathrm{Rp}-$} & \multicolumn{2}{|c|}{$\mathrm{Nrp}$} & \multicolumn{2}{|c|}{$\mathrm{Rp}$} \\
\hline & $M$ & $S E$ & $M$ & $S E$ & $M$ & $S E$ & $M$ & $S E$ & $M$ & $S E$ \\
\hline "P" & .73 & .03 & .04 & .03 & .05 & .02 & .02 & .01 & .02 & .00 \\
\hline “NP”' & .21 & .03 & .69 & .03 & .60 & .03 & .17 & .02 & .16 & .02 \\
\hline "New" & .06 & .01 & .27 & .02 & .35 & .02 & .80 & .02 & .82 & .02 \\
\hline
\end{tabular}

Note- $-\mathrm{Rp}+$, items from practiced categories that were individually practiced; Nrp, items from, or associated with, practiced categories; $\mathrm{Rp}-$, items from practiced categories that were not individually practiced; Rp, generally items associated with practiced categories; "P," claim that items were studied and practiced; "NP," claim that items were studied but not practiced. 
coding context may have helped to circumvent any potential effect of retrieval-induced forgetting.

Koutstaal et al.'s (1999) finding of retrieval-induced forgetting in recall, but not in recognition, is apparently consistent with the list-strength literature. For example, Ratcliff et al. (1990) showed that selectively strengthening some items in a list increases the probability of recalling those items, but decreases the probability of recalling nonstrengthened items. However, this strengthening does not adversely affect nonstrengthened items in tests of recognition. Our own results appear to be inconsistent with the list-strength literature in that strengthening items by virtue of retrieval practice indeed affects the later recognition of Rp - items. Perhaps the critical variable is whether the strengthening occurs because the exposure of some studied items is increased or because those items are actively retrieved. Although both methods produce facilitation of "strengthened" items, simple ex posure does not impair recognition of nonstrengthened items whereas retrieval practice does (e.g., Anderson, E. L. Bjork, \& R. A. Bjork, 2000; Ciranni \& Shimamura, 1999). Our results are also not entirely consistent with the part-set cuing and directed-forgetting literatures briefly mentioned earlier. Directed forgetting has been found in tests of recognition, but those effects are found only with an item-by-item forget instruction (see MacLeod, 1999, for details).

Because retrieval-induced forgetting has been found in tests of recall and recognition, Koutstaal et al.'s (1999) hypothesis that the consequence of retrieval practice is to impair conscious access to episodic retrieval of $\mathrm{Rp}-$ items becomes salient. What may be important is not whether the test presents item-specific cues, but whether the test is direct or indirect (cf. Butler et al., 2001). One way to simultaneously test these hypotheses would be to orthogonally manipulate direct versus indirect and itemspecific versus non-item-specific retrieval contexts. For example, to explore the role of item-specific cues tests of indirect memory such as category exemplar generation and free association could be compared with item-specific tests such as fragment completion. A comparison of direct versus indirect retrieval contex ts would test the implication from Koutstaal et al. that conscious access to memory is a critical variable.

We found in two experiments that retrieval-induced forgetting occurs in tests of item recognition. This finding is important because similar forms of retrieval interference (e.g., directed forgetting, list-strength effects) are difficult to obtain in recognition tests (Anderson \& R. A. Bjork, 1994). The hypothesis that retrievalinduced forgetting will be difficult to find when itemspecific retrieval cues are available (Butler et al., 2001) was not supported. We argue that any direct test of memory may reveal the detrimental effects of retrievalinduced forgetting. Future research exploring the direct/ indirect test variable may help disambiguate whether retrieval-induced forgetting affects either an item's representation per se (Anderson et al., 1994) or episodic access to that item (Koutstaal et al., 1999). We also believe that using tests of recognition allows researchers methodological flexibility and control. Perhaps researchers can use these benefits to explore other ways in which retrieval of information from memory affects associated, but nonretrieved, concepts.

\section{REFERENCES}

Anderson, M. C. (2003). Rethinking interference theory: Executive control and the mechanisms of forgetting. Journal of Memory \& Language, 49, 415-445.

Anderson, M. C., BJork, E. L., \& BJork, R. A. (2000). Retrieval-induced forgetting: Evidence for a recall-specif ic mechanism. Psychonomic Bulletin \& Review, 7, 522-530.

ANDERSON, M. C., \& BJORK, R. A. (1994). Mechanisms of inhibition in long-term memory: A new taxonomy. In D. Dagenbach and T. H. Carr (Eds.), Inhibitory processes in attention, memory, and language (pp. 265-325). San Diego: Academic Press.

ANDERSon, M. C., BJork, R. A., \& BJorK, E. L. (1994). Remembering can cause forgetting: Retrieval dynamics in long-term memory. Journal of Experimental Psychology: Learning, Memory, \& Cognition, 20, 1063-1087.

Anderson, M. C., De KoK, D., \& CHILd, C. (1997, November). Retrievalinduced forgetting on a test of recognition memory. Poster session presented at the Annual Meeting of the Psychonomic Society, Philadelphia.

Anderson, M. C., \& Spellman, B. A. (1995). On the status of inhibitory mechanisms in cognition: Memory retrieval as a model case. Psychological Review, 102, 68-100.

Battig, W. F., \& Montague, W. E. (1969). Category norms for verbal items in 56 categories: A replication and extension of the Connecticut norms. Journal of Experimental Psychology Monographs, 80(3, Pt. 2), 1-46.

Butler, K. M., Williams, C. C., Zacks, R. T., \& MAKi, R. H. (2001). A limit on retrieval-induced forgetting. Journal of Experimental Psychology: Learning, Memory, \& Cognition, 27, 1314-1319.

Cedrus Corporation (1999). SuperLab Pro for Windows (Version 2.0) [Computer software]. San Pedro, CA: Author.

Ciranni, M. A., \& Shimamura, A. P. (1999). Retrieval-induced forgetting in episodic memory. Journal of Experimental Psychology: Learning, Memory, \& Cognition, 25, 1403-1414.

Koutstaal, W., Schacter, D. L., Johnson, M. K., \& Galluccio, L. (1999). Facilitation and impairment of event memory produced by photograph review. Memory \& Cognition, 27, 478-493.

LANG, J. (2000). Retrieval failure due to response competition: Inhibition or interference? (Doctoral dissertation, Stanford University, 2000). Dissertation Abstracts International: Section B. The Sciences \& Engineering, 60(8-B), 4266.

LiNDSAY, D. S., \& JOHNSON, M. K. (1989). The eyewitness suggestibility effect and memory for source. Memory \& Cognition, 17, 349-358.

MACLEOD, C. M. (1999). The item and list methods of directed forgetting: Test differences and the role of demand characteristics. Psychonomic Bulletin \& Review, 6, 123-129.

MARSH, R. L., \& HICKS, J. L. (1998). Test formats change sourcemonitoring decision processes. Journal of Experimental Psychology: Learning, Memory, \& Cognition, 24, 1137-1151.

MCGEOCH, J. A. (1942). The psychology of human learning. New York: Longmans, Green.

NeELy, J. H., SCHMidT, S. R., \& RoEdIGER, H. L., III (1983). Inhibition from related primes in recognition memory. Journal of Experimental Psychology: Learning, Memory, \& Cognition, 9, 196-211.

Postman, L., \& STARK, K. (1969). The role of response availability in transfer and interference. Journal of Experimental Psychology, 79, 168-177.

RAAIJMAKERS, J. G. W., \& SHIFFrin, R. M. (1981). Search of associative memory. Psychological Review, 88, 93-134.

RATClifF, R., ClARK, S. E., \& ShIFFrin, R. M. (1990). The list-strength effect: I. Data and discussion. Journal of Experimental Psychology: Learning, Memory, \& Cognition, 16, 163-178. 
RoEdIGE R, H. L., III (1974). Inhibiting effects of recall. Memory \& Cog nition, 2, 261-269.

SLAMECKA, N. J. (1975). Intralist cueing of recognition. Journal of Verbal Learning \& Verbal Behavior, 14, 630-637.

STARNS, J. J., \& HICKS, J. L. (in press). Episodic generation can cause semantic forgetting: Retrieval-induced forgetting of false memories. Memory \& Cognition.

Todres, A. K., \& Watkins, M. J. (1981). A part-set cuing effect in recognition memory. Journal of Experimental Psychology: Human Learning \& Memory, 7, 91-99.

WATKINS, M. J. (1978). Engrams as cuegrams and forgetting as cue-overload: A cueing approach to the structure of memory. In C. R. Puff (Ed.), The structure of memory (pp. 347-372). New York: Academic Press.

\section{NOTE}

1. We also examined recognition latencies for evidence of retrieval inhibition. Rp + items were recognized fastest $(M=855, S E=32)$, Nrp items next fastest $(M=1,009, S E=43)$, and Rp - items the slowest $(M=1,033, S E=48)\left[F(2,76)=24.79, M S_{\mathrm{e}}=14,665.30\right]$. However, the nominal difference in Nrp and $\mathrm{Rp}-$ items was not significantly different by pairwise comparison $[t(39)<1.0]$.

(Manuscript received November 30, 2002; revision accepted for publication December 18, 2002.) 